\title{
Celiac disease in children: increasing prevalence and changing clinical presentations
}

Hasan M. Isa, MBBCh, CABP ${ }^{1}$, Eman Farid, MBBch, MSc, PhD², Jaafar J. Makhlooq, MBBCh, CABP (Part 1) ${ }^{3}$, Afaf M. Mohamed, ABFP, MPH ${ }^{4}$, Jumana G. Al-Arayedh, MD ${ }^{5}$, Fawzeya A. Alahmed, MD ${ }^{6}$, Shima Medani, MBBS, MSC, ABMLI ${ }^{7}$

${ }^{1}$ Pediatric Department, Salmaniya Medical Complex, Arabian Gulf University, Manama, Bahrain; ${ }^{2}$ Pathology Department, Salmaniya Medical Complex, Arabian Gulf University, Manama, Bahrain; ${ }^{3}$ Pediatric Department, Salmaniya Medical Complex, Manama, Bahrain; ${ }^{4}$ Puplic Health Department, Ministry of Health, Manama, Bahrain; ${ }^{5}$ Pediatric Department, Salmaniya Medical Complex, Manama, Bahrain; ${ }^{6}$ Pediatric Department, Salmaniya Medical Complex, Manama, Bahrain; ${ }^{7}$ Pathology Department, Salmaniya Medical Complex, Manama, Bahrain

Background: Celiac disease (CD) is a chronic autoimmune enteropathy. It results from genetic predisposition and exposure to gluten-containing food. The prevalence and presentation of CD vary among populations.

Purpose: This study aimed to describe the prevalence and clinical characteristics of $\mathrm{CD}$ in children in Bahrain.

Methods: We retrospectively reviewed the medical records of children diagnosed with $\mathrm{CD}$ in the pediatric department, Salmaniya Medical Complex, Bahrain, in 1988-2018. Their clinical, biochemical, serological, and histopathological findings were documented. Adherence to the recommended gluten-free $\operatorname{diet}(\mathrm{GFD})$ was assessed.

Results: Of 86 patients with CD, 67 were included. The CD prevalence was $0.02 \%$. A significant increase in prevalence in the last decade was observed $(P<0.0001)$. Thirty-eight patients $(56.7 \%)$ were males. The median (interquartile range) age at presentation was $4.45(1.5-7.3)$ years. A family history of $\mathrm{CD}$ was positive in 13 out of 43 patients (30.2\%). Pallor and failure to thrive were the most common presentations. The most frequent associated disease was iron-deficiency anemia in 23 patients (69.7\%). Positive serology was found in 32 of 45 patients (71.1\%). Marsh-Oberhuber type III was found in 16 of 35 patients (45.7\%). Seropositive patients were significantly older $(P=0.025)$ and had more severe duodenal histology $(P=0.002)$. Adherence to GFD was poor in 27 patients $(64.3 \%)$. Conclusion: This study revealed a significant increase in $\mathrm{CD}$ prevalence over the last decade. Atypical presentations were frequent. Most patients had poor adherence to GFD.

Key words: Celiac disease, Child, Prevalence, Clinical presentation, Bahrain

\section{Key message}

Question: What are the prevalence and clinical characteristics of celiac disease $(\mathrm{CD})$ in children in Bahrain?
Finding: We found a significant increase in $\mathrm{CD}$ prevalence over the last decade $(P=0.0001)$. A male predominance was noted. Atypical presentations were common. Most patients had poor adherence to a gluten-free diet.

Meaning: $\mathrm{CD}$ is an underdiagnosed condition. Atypical symptoms should be considered to prevent missing patients with CD.

\section{Introduction}

Celiac disease $(\mathrm{CD})$ is a chronic autoimmune enteropathy. ${ }^{1-5)}$ It results from genetic predisposition and exposure to glutencontaining food. ${ }^{1,2,4,5)}$ Individuals carrying human leucocytes antigen (HLA) markers DQ2 or DQ8 are genetically predisposed. ${ }^{4-6)}$ Gluten is a protein found in wheat, rye, and barley; the main ingredients of bread, pasta, and pastries. ${ }^{2-4,7}$ Gluten works as a triggering factor for $\mathrm{CD}$, but the interaction between genetic and environmental factors is still not fully understood. ${ }^{4)}$

CD prevalence varies widely between populations to be anywhere between $0.5 \%$ and $1 \% .{ }^{8,9)}$ The classical symptoms of $\mathrm{CD}$ in children are chronic diarrhea, anemia, and failure to thrive (FTT) ${ }^{10)}$ However, not all the patients came with the classical presentations. Some may present with food allergy and later on found to have CD. ${ }^{11)}$ Identifying celiac-specific antibodies by serological tests has allowed us to detect asymptomatic, latent, and atypical cases. ${ }^{6,10)}$ The diagnosis of $\mathrm{CD}$ is confirmed by the presence of histopathological lesions including crypt hyperplasia, an increased intraepithelial lymphocyte (IEL) count (over 25 lymphocytes per 100 enterocytes), with or without villous atrophy within the small intestinal mucosa (Marsh classification type II or III as modified by Oberhuber). . $^{\text {) }}$

Though CD is a chronic multisystem disease, mucosal damage can be reversed, and antibodies titer drop remarkably if gluten is completely eliminated from the diet.6) Gluten-free

\footnotetext{
Corresponding author: Hasan M. Isa, MBBCh, CABP. Pediatric Department, Assistant professor, Arabian Gulf University, Villa 510, Road 2618, Barbar 526, manama, Bahrain, Manama, Bahrain 


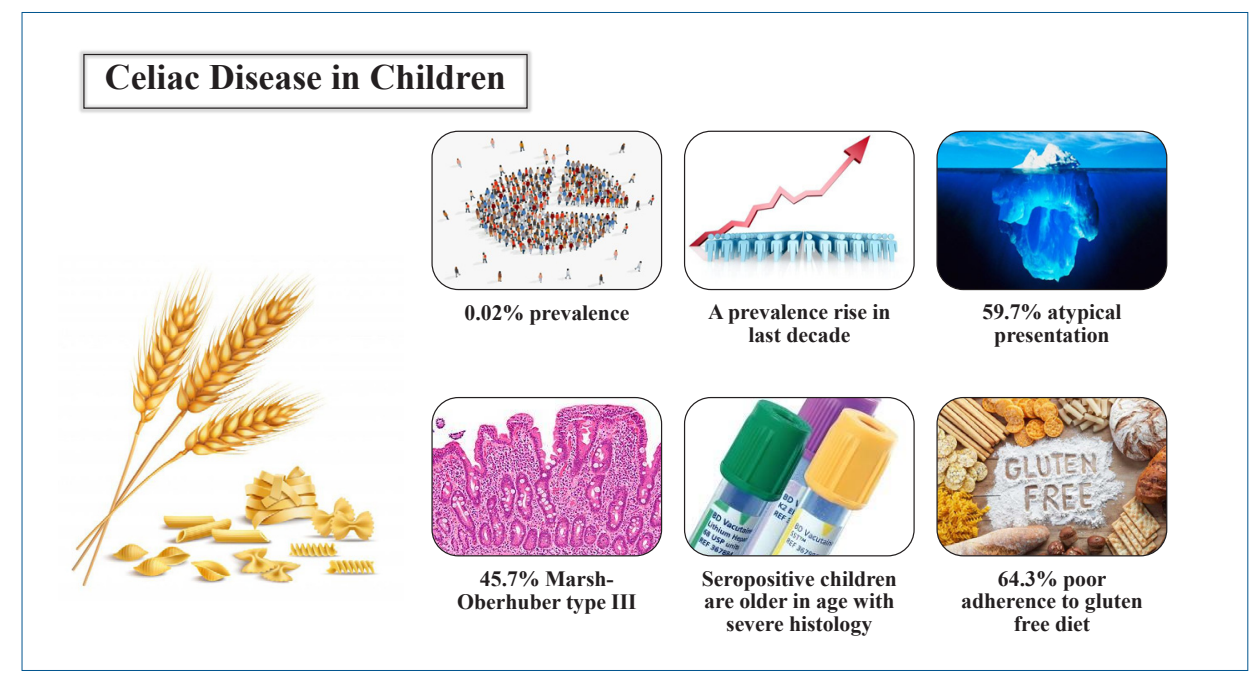

Graphical abstract. Prevalence and associated clinical characteristics of celiac disease in children in Bahrain.

diet (GFD) remains the treatment of choice in CD despite being costly, restraining, and adhering to it is difficult. ${ }^{3)}$ Recent spread of $\mathrm{CD}$ awareness encouraged food industries to produce ranges of harmless substitutes for gluten-containing foods, but the choices are still restricted. ${ }^{3)}$

$\mathrm{CD}$ in children has been evaluated worldwide and in several neighboring countries to Bahrain. ${ }^{1-7,10-21)}$ To our knowledge, CD in children has not been evaluated in Bahrain. This study aimed to describe the prevalence, clinical, laboratory, endoscopic, and histopathological characteristics of children with CD in Bahrain and to evaluate their adherence to GFD.

\section{Methods}

\section{Patients and materials}

This is a retrospective cross-sectional review of all medical records for patients with $\mathrm{CD}$ in the pediatric department, Salmaniya Medical Complex (SMC), Bahrain from January 1988 to November 2018. SMC is the only tertiary hospital in Bahrain and all pediatric patients suspected to have $C D$ are referred to, diagnosed, and managed in this hospital. Accordingly, prevalence and annual incidence of pediatric $\mathrm{CD}$ in Bahrain were calculated. Patients over 18 years were excluded. Patients were diagnosed to have $\mathrm{CD}$ based on a combination of clinical, serological, and histopathological findings. ${ }^{22)} \mathrm{A}$ combination of at least one positive CD-specific serological test like anti-tissue transglutaminase (anti-tTG) antibodies, antiendomy. sial antibodies (AEA), or antigliadin antibodies (AGA) along with histologic changes of modified Marsh grade II or more on the small intestinal biopsies is required to diagnose $\mathrm{CD} .{ }^{23)} \mathrm{In}$ the absence of serological data, a combination of modified Marsh grade II or more and clinical and/or histological improvements after starting the patient on a GFD are also accepted as a diagnosis criteria. ${ }^{23)}$

Sociodemographic data were collected including gender, nationality, age at presentation, age at diagnosis, year of presen- tation, gestational age, delivery type, birth weight, breastfeeding history, time of cereal introduction, and family history of CD. Clinical presentations including pallor, FTT, abdominal distension, short stature, abdominal pain, diarrhea, constipation, and vomiting were collected. Data about the presence of associated diseases were also collected. Laboratory investigations including complete blood count, liver function tests, thyroid function tests, serum iron, ferritin, calcium, phosphorus, and immunoglobulin A (IgA) levels were collected. Anemia was defined as hemoglobin level $<11 \mathrm{~g} / \mathrm{dL}$ in children 6 months to 5 years old, $<11.5 \mathrm{~g} / \mathrm{dL}$ in children 5 to 11 years old, $<12 \mathrm{~g} /$ $\mathrm{dL}$ in children 12 to 13 years old, $<13 \mathrm{~g} / \mathrm{dL}$ in men, and $<12$ $\mathrm{g} / \mathrm{dL}$ in nonpregnant women. ${ }^{24)}$ Iron-deficiency anemia (IDA) was diagnosed based on low serum iron levels with or without low serum ferritin levels. $\left.{ }^{25}\right) \mathrm{CD}$-specific antibodies including Anti-tTG, AEA, AGA, and HLA genotyping were recorded. Endoscopic and histological findings of the small intestine biopsies were also collected. Immuno-histochemical staining for cluster of differentiation 3 (CD3) was recorded. Outpatient follow-up period was calculated. The weight and length/height of the last outpatient visit were collected and body mass index (BMI) [weight $(\mathrm{kg}) /$ height $\left.^{2}(\mathrm{~m})\right]$ was calculated. These growth parameters were presented as a standard deviation (SD) from age- and sex-specific reference means, and they were plotted on the relevant World Health Organization (WHO) charts. The WHO standards and references were used to assess the nutritional status. Accordingly, FTT (wasting) and risk of overweight were defined as BMI for age $<-2$ and $>+1 \mathrm{SD}$, respectively while stunting and severe stunting were defined as length/heightfor-age $<-2$ and $<-3 \mathrm{SD}$, respectively. ${ }^{26,27)}$ Adherence to GFD was evaluated via reviewing the dietary notes of each outpatient clinic visit.

Based on serological test results, the patients were divided into 2 groups (seropositive and seronegative). The 2 groups were compared according to the age at presentation, gender, clinical presentations, BMI, weight status, height status, presence of associated diseases, hemoglobin levels, Marsh-Oberhuber 
classifications, and adherence to GFD.

\section{Statistical analysis}

Patient's data were analyzed using IBM SPSS Statistics ver. 21.0 (IBM Co., Armonk, NY, USA) program. Data were presented as frequency and percentage for categorical variables or mean and SD or median and interquartile range (IQR) for continuous variables. $\mathrm{CD}$ incidence and prevalence were calculated after exclusion of non-Bahraini patients. Kruskal-Wallis test was used to compare CD prevalence in the last 3 decades. To compare the 2 serological groups, Pearson chi-square test or Fisher exact test were used for categorical variables. Mann-Whitney $U$ test or Student $t$ tests were used for continuous variables. A 2 -sided $P$ value of $<0.05$ was considered statistically significant.

\section{Results}

During the study period, 86 patients were diagnosed to have CD. Nineteen patients were excluded (10 patients were more than 18 years of age and 9 patients with unknown age at the time of presentation). The remaining 67 patients were analyzed. According to 2018 Bahrain health statistics, the population of Bahrain is 1,503,091, with 347,886 people aged less than 18 years (277,679 Bahraini and 97,207 non-Bahraini). After exclusion of non-Bahraini patients, the median annual incidence was 0.45 per 100,000 per year (IQR, 0.0-1.2). The annual incidence of CD among Bahraini and non-Bahraini children is shown in Fig. 1. On comparing the numbers of the 48 Bahraini patients in the last 3 decades, 4 patients $(8.3 \%)$ were diagnosed between 1989 and 1998 (164,470 population at risk), 3 patients
(6.3\%) between 1999 and 2008 (227,110 population at risk), and 41 patients (85.4\%) between 2009 and 2018 (277,679 population at risk). The overall prevalence of $\mathrm{CD}$ among Bahraini children is $0.018 \%$ (18.36 patients per 100,000). The median (IQR) prevalence of CD in Bahraini children in each decade was $0 \%(0.0-0.0006), 0 \%(0.0-0.00046)$, and $0.0012 \%$ (0.001-0.0023), respectively. There was a significant increase in the prevalence of CD over the last 3 decades $(P<0.0001)$.

Demographic data are shown in Table 1 . Thirty-eight patients (56.7\%) were males, and 29 (43.3\%) were females. Fifty-one patients (76.1\%) were Bahraini, and 16 (23.9\%) were nonBahraini (5 patients were from Pakistan, 3 from Egypt, 2 from Syria, 2 from India, 1 from each Saudi Arabia, Yemen, United Arab Emirates, and Algeria). Median (IQR) age at the time of the study was 8.8 (6.4-14.2) years. Family history of CD was found in 13 out of 43 patients (30.2\%); 11 (25.6\%) had first degree while 2 had second-degree relatives.

Out of 63 patients $(94 \%)$ with available detailed clinical history, 58 patients $(92.1 \%)$ were symptomatic at the time of presentation while 5 (7.9\%) were asymptomatic and they were detected via CD screening (3 patients had first degree relative with $\mathrm{CD}, 1$ with autism, and 1 with type 1 diabetes mellitus). The commonest clinical presentations were pallor, FTT, and abdominal distension (Table 2).

Forty-one out of 65 patients (63.1\%) had at least one associated disease (Table 3). The most frequent associated diseases were IDA in 23 patients (69.7\%), followed by a history of food allergy in 16 patients (25.4\%); 5 of them had multiple food allergies. Dairy product allergy was reported in 6 patients, egg allergy in 4, nut allergy in 3, orange allergy in 2, wheat allergy in 2 while allergy to tomato, seafood, kiwi, lemon, mango, sesame,

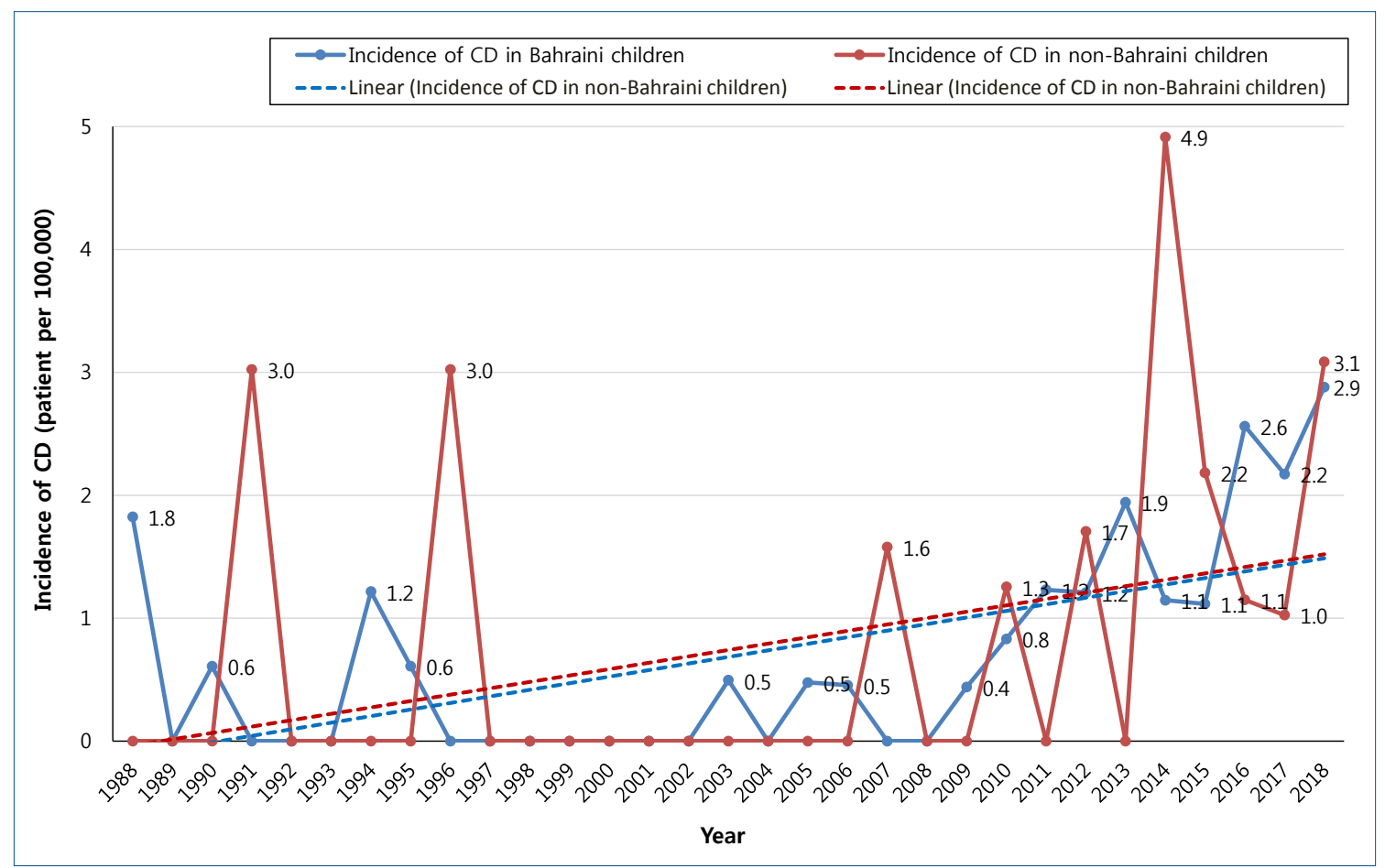

Fig. 1. Incidence of celiac disease in Bahraini versus non-Bahraini children, 1998-2018. 
Table 1. Demographic data of 67 pediatric patients with celiac disease

\begin{tabular}{|c|c|}
\hline Variable & Value \\
\hline \multicolumn{2}{|l|}{$\operatorname{Sex}(n=67)$} \\
\hline Male & $38(56.7)$ \\
\hline Female & $29(43.3)$ \\
\hline \multicolumn{2}{|l|}{ Nationality $(n=67)$} \\
\hline Bahraini & $51(76.1)$ \\
\hline Non-Bahraini & $16(23.9)$ \\
\hline \multicolumn{2}{|l|}{ Gestational age $(n=42)$} \\
\hline Term & $41(97.6)$ \\
\hline Preterm & $1.0(2.4)$ \\
\hline \multicolumn{2}{|l|}{ Type of delivery $(n=42)$} \\
\hline Normal vaginal delivery & $33(78.6)$ \\
\hline Lower segment caesarian section & $9(21.4)$ \\
\hline Birth weight $(\mathrm{kg})(\mathrm{n}=36)$ & $3.08 \pm 0.56$ \\
\hline History of breastfeeding ( $n=26)$ & $21(80.8)$ \\
\hline Time of cereal introduction (mo), median (IQR) & $6.0(5.0-8.3)$ \\
\hline Age at presentation (yr), ( $n=62)$ & $4.45(1.5-7.3)$ \\
\hline \multicolumn{2}{|l|}{ Presentation age category (yr) (n=62) } \\
\hline $0-4$ & $33(53.2)$ \\
\hline $5-9$ & $19(30.6)$ \\
\hline $10-14$ & $8.0(12.9)$ \\
\hline $15-18$ & $2.0(3.2)$ \\
\hline Age at diagnosis (yr) $(n=52)$ & $3.65(1.0-6.8)$ \\
\hline Age at time of study (yr) (n=66) & $8.8(6.4-14.2)$ \\
\hline \multicolumn{2}{|l|}{ Age at time of study category $(y r)(n=67)$} \\
\hline $0-4$ & $8(11.9)$ \\
\hline $5-9$ & $29(43.3)$ \\
\hline $10-14$ & $14(20.9)$ \\
\hline $15-18$ & $4(6)$ \\
\hline$>18$ & $12(17.9)$ \\
\hline Family history of celiac disease $(n=43)$ & $13(30.2)$ \\
\hline
\end{tabular}

Values are presented as number (\%), mean \pm standard deviation, or median (interquartile range).

$\mathrm{SD}$, standard deviation; IQR, interquartile range.

Table 2. Clinical presentations of pediatric patients with celiac disease $(n=67)$

\begin{tabular}{lc}
\hline Clinical presentation & No. of patients (\%) \\
\hline Symptomatic & $58(86.6)$ \\
Pallor & $40(59.7)$ \\
Failure to thrive & $37(55.2)$ \\
Abdominal distension & $35(52.2)$ \\
Short stature & $35(52.2)$ \\
Chronic abdominal pain & $30(44.8)$ \\
Diarrhea & $28(41.8)$ \\
Constipation & $21(31.3)$ \\
Vomiting & $18(26.9)$ \\
Chronic fatigue & $17(25.4)$ \\
Muscular hypotrophy & $8.0(11.9)$ \\
Mouth ulcers & $8.0(11.9)$ \\
Dermatitis herpetiformis & $3.0(4.5)$ \\
Edema & $2.0(3.0)$ \\
Clubbing & $2.0(3.0)$ \\
Asymptomatic & $5.0(7.5)$ \\
Missing data & $4.0(6.0)$
\end{tabular}

Table 3. Associated diseases in 67 pediatric patients with celiac disease

\begin{tabular}{lc}
\hline Associated disease & Patient/total, n (\%) \\
\hline Iron-deficiency anemia & $23 / 33(69.7)$ \\
\hline History of food allergy & $16 / 63(25.4)$ \\
Glucose 6 phosphate dehydrogenase reduced activity & $12 / 65(18.5)$ \\
Bronchial asthma & $12 / 65(18.5)$ \\
Type 1diabetes mellitus & $9.0 / 65(13.8)$ \\
Hypothyroid & $7.0 / 59(11.9)$ \\
Vitamin D deficiency & $6.0 / 65(9.2)$ \\
Rickets & $5.0 / 64(7.8)$ \\
Gastroesophageal reflux disease & $5.0 / 64(7.8)$ \\
Selective immunoglobulin A deficiency & $4.0 / 39(10.3)$ \\
Hearing loss & $4.0 / 64(6.3)$ \\
Autism & $4.0 / 65(6.2)$ \\
Eosinophilic esophagitis & $3.0 / 65(4.6)$ \\
Congenital heart disease & $2.0 / 65(3.1)$ \\
Lactose intolerance & $2.0 / 65(3.1)$ \\
Other diseases & $16 / 65(24.6)$
\end{tabular}

a)Trisomy 21, epilepsy and Panayiotopoulos syndrome, developmental delay, alopecia areata, eczema, epigastric hemangioma, nasal septal deviation, recurrent otitis media, beta thalassemia minor, thrombocytopenia, myeloproliferative disorder, biliary atresia, cystic fibrosis, Meckel's diverticulum, and arthritis in 1 patient each.

and antibiotics each in 1 patient. One patient had eosinophilic gastroenteritis and hearing loss.

Results of laboratory investigations at the initial presentation are shown in Table 4. Leukocytosis was found in 25.5\% (14 of 55 patients). Leukopenia was found in 1 patient (1.8\%). Anemia was found in $51.8 \%$ ( 29 of 56 patients) and low serum iron levels in $69.7 \%$ (23 of 34 patients). Thrombocytosis was found in $28.3 \%$ (15 of 53 patients). Hypoproteinemia was found in $13.9 \%$ (5 of 37 patients) while hypoalbuminemia was found in $11.9 \%$ (5 of 42 patients) and hyperglobulinemia in 27\% (10 of 37 patients). One patient (1.7\%) had low serum globulin. Elevated liver enzymes were noted in 30.9\% (13 of 42 patients) with high alanine aminotransferases (ALT) in 10 patients (23.8\%). Hypocalcemia was found in $37.8 \%$ (14 of 37 patients). Serum phosphorus and ferritin were normal in all tested patients; 14 (20.9\%) and 35 patients (52.2\%); respectively.

Positive CD serology was seen in 32 patients (71.1\%) out of 45 (67.2\%) with available serological records. Positive AGAIgG, AGA-IgA, anti-EMA antibodies, and tTG-IgA antibodies were found in $64.1 \%$ (25 of 39 patients), $48.4 \%$ (15 of 31 patients), $44.8 \%$ ( 13 of 29 patients), and $29 \%$ (9 of 31 patients); respectively. All the 8 tested patients for HLA class II types were positive (6 patients for DQ2 alone, 1 for DQ8 alone, and 1 for both DQ2 and DQ8).

All the patients had small intestinal biopsies collected, 63 biopsies (94\%) were collected via upper intestinal endoscopy while $4(6 \%)$ via Crosby-Kugler capsules. Out of the 67 patients, 25 (37.3\%) had endoscopic data and 35 (52.2\%) had histology data available (Table 5). Out of the 25 patients with endoscopic data, 9 (36\%), 14 (56\%), and 16 patients (64\%) had positive findings in esophageal, gastric, and duodenal endoscopies; 
Table 4. Results of laboratory investigations at presentation of 67 pediatric patients with celiac disease

\begin{tabular}{lcc}
\hline Laboratory investigation & Value & $\begin{array}{c}\text { Normal } \\
\text { range }\end{array}$ \\
\hline White blood cells count $\left(\times 10^{9} / \mathrm{L}\right)(\mathrm{n}=55)$ & $8.0(6.0-10)$ & $3.6-9.6$ \\
Hemoglobin $(\mathrm{g} / \mathrm{dL})^{\mathrm{a})}(\mathrm{n}=56)$ & $11.7 \pm 1.47$ & $12-14.5$ \\
Platelets $\left(\times 10^{9} / \mathrm{L}\right)(\mathrm{n}=53)$ & $315(259-449)$ & $140-440$ \\
Protein $(\mathrm{g} / \mathrm{L})(\mathrm{n}=37)$ & $71.4 \pm 6.7$ & $64-82$ \\
Albumin $(\mathrm{g} / \mathrm{L})(\mathrm{n}=42)$ & $41 \pm 4.9$ & $35-50$ \\
Globulin $(\mathrm{g} / \mathrm{L})(\mathrm{n}=37)$ & $29.6 \pm 5.7$ & $15-30$ \\
Bilirubin $(\mu \mathrm{mol} / \mathrm{L})(\mathrm{n}=41)$ & $5.0(4.0-7.4)$ & $<18$ \\
Alanine aminotransferase $(\mu / \mathrm{L})(\mathrm{n}=42)$ & $25(16-41)$ & $<35$ \\
Alkaline phosphatase $(\mu / \mathrm{L})(\mathrm{n}=41)$ & $198(141-270)$ & $150-420$ \\
Gamma glutamyltransferase $(\mu / \mathrm{L})(\mathrm{n}=41)$ & $14(10-18)$ & $<35$ \\
Serum iron $(\mu \mathrm{mol} / \mathrm{L})(\mathrm{n}=34)$ & $8.0(5.5-12.5)$ & $9-30.4$ \\
Serum calcium $(\mathrm{mmol} / \mathrm{L})(\mathrm{n}=37)$ & $2.29 \pm 0.16$ & $2.2-2.7$ \\
Serum IgA level $(\mathrm{g} / \mathrm{L})(\mathrm{n}=39)$ & $1.13(0.8-1.5)$ & $0.5-4$ \\
AGA-IgG antibodies $(\mu / \mathrm{mL})(\mathrm{n}=39)$ & $25(6.4-81)$ & $<10$ \\
AGA-IgA antibodies $(\mu / \mathrm{mL})(\mathrm{n}=31)$ & $16(1.7-46.4)$ & $<10$ \\
Positive anti-EMA antibodies $(\mathrm{n}=29)$ & $13(44.8)$ & Negative \\
TTG-IgA antibodies $(\mu / \mathrm{mL})(n=31)$ & $31.6(3.8-101.4)$ & $<10$ \\
\hline
\end{tabular}

Values are presented as median (interquartile range) or mean \pm standard deviation.

$I Q R$, interquartile range; SD, standard deviation; $A G A-I g G$, anti-gliadinantibodies immunoglobulin G; AGA-IgA, anti-gliadin-antibodies immunoglobulin A; EMA, antiendomysial immunoglobulin A; TTG-IgA, tissue transglutaminase immunoglobulin $A$.

a) Anemia was defined as hemoglobin level $<11 \mathrm{~g} / \mathrm{dL}$ in children 6 months to 5 years old, $<11.5 \mathrm{~g} / \mathrm{dL}$ in children 5 to 11 years old, $<12 \mathrm{~g} / \mathrm{dL}$ in children 12 to 13 years old, $<13 \mathrm{~g} / \mathrm{dL}$ in men, and $<12 \mathrm{~g} / \mathrm{dL}$ in nonpregnant women.

respectively. Some patients had more than one finding. Findings suggestive of $\mathrm{CD}$ (scalloping, shining, and flattening of duodenal mucosa) were noted in 12 patients (48\%).

In terms of histological reports, 27 patients $(40.3 \%)$ had esophageal and gastric biopsy reports available while 35 patients (52.3\%) had available duodenal biopsy reports. Out of 35 patients with duodenal reports, 18 (51.4\%) had histological findings suggestive of CD (Marsh II or III). Marsh III was seen in 16 patients (45.7\%) with CD histological findings. IEL were detected in 51.4\% (18 of 35). Immuno-histochemical staining for $\mathrm{CD} 3$ showed increase in IEL in the all 3 tested patients. Out of 31 patients with available antral biopsy cultures, 2 patients $(6.5 \%)$ were positive for helicobacter pylori.

The median (IQR) follow-up period was 9.3 years (6.4-21). Three patients (4.5\%) died, 1 passed away secondary to an associated myeloproliferative disease while the other 2 patients from Saudi Arabia and United Arab Emirates died out of Bahrain with unclear cause. According to the last outpatient visit anthropometric data, weight was available for 47 patients (70.2\%) and height for 44 (56.7\%). Thirty-seven patients $(78.7 \%)$ had normal weight, 8 patients (17\%) were wasted, and 2 (4.3\%) were at risk of overweight. In terms of height, $38(86.4 \%)$ had normal height, 5 (11.4\%) were stunted, and 1 (2.3\%) was severely stunted. Adherence to GFD was strict in 15 (45.7\%) and poor in $27(64.3 \%)$ out of 42 patients (62.7\%) with available data.

Comparison between children with $\mathrm{CD}$ according to the
Table 5. Endoscopic and histopathological findings of patients with celiac disease

\begin{tabular}{lc}
\hline Investigation & No. of patients (\%) \\
\hline Endoscopic findings & $25(37.3)$ \\
Esophagus & \\
Normal & $16(64.0)$ \\
White dot disease & $4(16.0)$ \\
Distal erythema & $4(16.0)$ \\
Longitudinal ulcers & $3(12.0)$ \\
Hiatal hernia & $1(4.0)$ \\
Stomach & \\
Normal & $11(44.0)$ \\
Erythema & $9(36.0)$ \\
Nodularity & $5(20.0)$ \\
Ulcers & $1(4.0)$ \\
Hemorrhagic spots & $1(4.0)$ \\
Pancreatic nest & $1(4.0)$ \\
Duodenum & \\
Normal & $9(36.0)$ \\
Shining/flat mucosa & $10(40.0)$ \\
Scalloping mucosa & $7(28.0)$ \\
Erythema & $4(16.0)$ \\
Ulcers & $2(8.0)$ \\
Edema & $1(4.0)$ \\
Histopathological findings & $27(40.3)$ \\
Esophagus & \\
Normal & $15(22.8)$ \\
Eosinophilic esophagitis & $15(55.6)$ \\
Papillary hyperplasia & $4(14.8)$ \\
Mild chronic esophagitis & $3(11.1)$ \\
Reflux esophagitis & $2(7.4)$ \\
Lymphocytes in basal region & $2(7.4)$ \\
Mormalinal & $1(3.7)$ \\
\hline
\end{tabular}

serological tests results is shown in Table 6. Patients with positive CD serology were significantly older in age $(P=0.025)$ and had more severe duodenal histology based on Marsh-Oberhuber classification $(P=0.002)$. There was no significant difference found between seropositive and seronegative patients in regard to sex, clinical presentations, BMI, weight status, height status, presence of associated diseases, hemoglobin levels, or adherence to GFD. 
Table 6. Comparison of children with celiac disease according to serological tests results

\begin{tabular}{|c|c|c|c|}
\hline \multirow{2}{*}{ Variable } & \multicolumn{2}{|c|}{ Celiac disease serology $(n=45)$} & \multirow{2}{*}{$P$ value $^{\text {a) }}$} \\
\hline & Positive, 32 (71.1\%) & Negative, 13 (28.9\%) & \\
\hline Age at presentation $(y r)(n=41)$ & $6.3 \pm 4.3$ & $3.1 \pm 2.5$ & 0.025 \\
\hline \multicolumn{4}{|l|}{$\operatorname{Sex}(n=45)$} \\
\hline Female & $19(59.4)$ & $5.0(38.5)$ & 0.323 \\
\hline Male & $13(40.6)$ & $8.0(61.5)$ & \\
\hline \multicolumn{4}{|l|}{ Clinical presentation $(n=44)$} \\
\hline Symptomatic & $30(93.8)$ & $11(84.6)$ & 0.204 \\
\hline Asymptomatic & $1.0(3.1)$ & $2.0(15.4)$ & \\
\hline Body mass index $\left(\mathrm{kg} / \mathrm{m}^{2}\right)(\mathrm{n}=32)$ & $16 \pm 3.0$ & $15 \pm 3.0$ & 0.503 \\
\hline \multicolumn{4}{|l|}{ Weight status ( $n=35)$} \\
\hline Normal & $23(71.9)$ & $4.0(30.8)$ & 0.224 \\
\hline Wasted & $4.0(12.5)$ & $3.0(23.1)$ & \\
\hline Risk of overweight & $1.0(3.1)$ & $0.0(0.0)$ & \\
\hline \multicolumn{4}{|l|}{ Height status $(n=33)$} \\
\hline Normal & $22(68.8)$ & $6.0(46.2)$ & 1.000 \\
\hline Stunted & $4.0(12.5)$ & $1.0(7.7)$ & \\
\hline \multicolumn{4}{|l|}{ Associated disease $(n=44)$} \\
\hline Yes & $27(84.4)$ & $10(76.9)$ & 0.404 \\
\hline No & $4.0(12.5)$ & $3.0(23.1)$ & \\
\hline Hemoglobin level (g/dL) $(n=41)$ & $11.5 \pm 1.13$ & $11.1 \pm 1.37$ & $0.307(-1.24 \text { to } 0.40)^{b)}$ \\
\hline \multicolumn{4}{|l|}{ Marsh-Oberhuber classification ( $\mathrm{n}=27$ ) } \\
\hline M O=normal & $1.0(3.1)$ & $4.0(30.8)$ & 0.002 \\
\hline M I=increased intraepithelial lymphocytes & $6.0(18.8)$ & $1.0(7.7)$ & \\
\hline M II=increased intraepithelial lymphocytes & $2.0(6.3)$ & $0.0(0.0)$ & \\
\hline$M \| I^{c)}$ & $13(40.6)$ & $0.0(0.0)$ & \\
\hline \multicolumn{4}{|l|}{ Adherence to gluten-free diet $(n=35)$} \\
\hline Poor & $14(43.8)$ & $8.0(61.5)$ & 0.259 \\
\hline Strict & $11(34.4)$ & $2.0(15.4)$ & \\
\hline
\end{tabular}

Values are presented as mean \pm standard deviation or number (\%).

a)Pearson chi-square or Fisher exact test was used for categorical variables, while Mann-Whitney $U$ and Student $t$ test was used for continuous variables. ${ }^{\text {b) }} 95 \%$ confidence interval. ${ }^{c} \mathrm{M} \| \mathrm{ll} a=$ partial villus atrophy; $\mathrm{M}$ IIIb=subtotal villus atrophy; $\mathrm{M}$ IIIc=total villus atrophy.

Boldface indicates a statistically significant difference with $P<0.05$.

\section{Discussion}

The present study showed an overall prevalence of $\mathrm{CD}$ in children from Bahrain of $0.02 \%$ with a significant increase in the prevalence during the last 3 decades. However, CD prevalence in the present study is lower than that reported globally where prevalence of $\mathrm{CD}$ is ranging between $0.7 \%$ and $1.4 \% .6,7,9,28)$ Globally, CD prevalence has also increased overtime from $0.6 \%$ (1991-2000) to $0.8 \%$ (2001-2016). ${ }^{28)}$ Moreover, a study from United Kingdom reported that the prevalence of CD among children older than 2 years had tripled over the 20 years between 1993 and 2012. ${ }^{13)}$ Similar to our study, most of the patients with CD from Kuwait were diagnosed in the second half of the study period (between 1998 and 2010). ${ }^{14)}$

In this study, the estimated median annual incidence of $\mathrm{CD}$ in children is 0.45 per 100,000 people per year (IQR, 0.0-1.2). This incidence is also low when compared to studies from Italy and United Kingdom which reported incidence of 11.9 and 11.8 per 100,000 person-years; respectively. ${ }^{12,13)}$ This variation in the prevalence and incidence of $\mathrm{CD}$ between different countries can be attributed to vague presentations and delayed diagnosis in one hand; and to increased number of screened high-risk groups and/ or increased awareness among physicians about $\mathrm{CD}$ on the other hand. $6,14,29,30)$

$\mathrm{CD}$ tends to manifest more in children aged $0-4$ years. ${ }^{12)}$ The current study showed that 33 patients (53.2\%) presented before the age of 4 years followed by a decline in the number reaching only 2 patients (3.2\%) between the age of 15 and 18 years. This decrease in incidence with age is also shown by studies from India and Italy. ${ }^{10,12)}$ Despite the fact that CD is considered an infantile disease, diagnosis in later childhood is not uncommon. ${ }^{7,10)}$ There is an upward shift of age at diagnosis of the disease due to the change in the clinical presentations from malabsorption syndrome to a milder form. ${ }^{2,29)}$

The current study showed more male patients $(n=38,56.7 \%)$ affected with CD compared to 29 females (43.3\%). This finding is in contrary to what has been consistent in several studies of different populations where a higher incidence in females was noticed. ${ }^{1-3,5,6,10-15,17-19,28)}$ The present study also showed a positive family history of CD in 13 (30.2\%). Likewise, studies 
from Saudi Arabia and Finland revealed positive family history in 29.2\% and 26.4\%; respectively. ${ }^{3,15}$ However, Kuwait study reported affected first degree relatives in only $9 \%{ }^{14)}$

$\mathrm{CD}$ can present with either typical (symptomatic) or atypical (asymptomatic or screened) presentations. ${ }^{9}$ In the current study, 58 patients $(86.6 \%)$ were symptomatic while $5(7.5 \%)$ were asymptomatic. Similarly, studies from Saudi Arabia and Italy showed a majority of symptomatic patients of $92 \%$ and $60.4 \%$, respectively. ${ }^{3,12)}$ Younger age at presentation is considered a consistent finding of typical disease presentation while children with atypical disease presenting later in life. ${ }^{2,11)}$ This could be owed to the fact that younger patients present with classical symptoms such as diarrhea, FTT, abdominal distension, and anemia which are easy to recognize. ${ }^{2,7,10,11,14)}$ On the other hand, older children happened to complain of vague symptoms and often missing any gastrointestinal (GI) symptoms. ${ }^{11)}$

$\mathrm{CD}$ has a broad spectrum of clinical presentations. ${ }^{14)}$ Moreover, changes in the $\mathrm{CD}$ clinical presentation have become obvious since $80 \mathrm{~s}^{30}{ }^{30}$ Extraintestinal symptoms such as anemia, short stature, bone, hepatic and neurological issues have become more recognized. ${ }^{30)} \mathrm{CD}$ in children may be considered a systemic disease as extraintestinal manifestations dominate $\mathrm{CD}$ presentations of more than half of patients. ${ }^{30)}$ In the present study, the commonest presentations were pallor, FTT, abdominal distention, short stature, and chronic abdominal pain. However, a prospective study from India showed that diarrhea, FTT, and abdominal distension are the major symptoms of the disease while abdominal pain, vomiting, and constipation are relatively less common. ${ }^{10)}$ In contrary, a study from Saudi Arabia showed that the commonest presentation was chronic abdominal pain followed by poor weight gain. ${ }^{3)}$ Yet, recurrent mild abdominal pain is a common complaint in patients who were diagnosed upon screening. ${ }^{14)}$

Pallor is considered as one of the atypical presentations of CD. ${ }^{14)}$ However, it was the commonest presentation in this study and was found in 40 patients (59.7\%). Similarly, pallor was found in 15 out 25 symptomatic patients (60\%) from Kuwait. ${ }^{14)}$ Moreover, pallor was seen in $100 \%$ of 134 patients from India. ${ }^{10)}$ Conversely, studies from Saudi Arabia and Turkey reported anemia as a clinical presentation in only $12.4 \%$ and 5.1\% patients; respectively. ${ }^{1,3)}$

While FTT was the most frequent symptom of patients from Turkey (81.4\%) and Kuwait (72\%), the current study demonstrated FTT in 37 patients (55.2\%), being the second commonest clinical presentation. ${ }^{2,14)}$ Similarly, Saudi and Indian studies reported FTT in 54\% and 52.2\%, respectively, as a second most common presentation. ${ }^{3,10)}$ A retrospective study from Finland looked into the etiology of decreased growth in 530 patients with $\mathrm{CD}$ found that an earlier age of onset, severe clinical, serological, and histological finding are associated with disturbed growth at CD diagnosis. ${ }^{15)}$ Despite that FTT is a common complain, Kuwaiti study documented patients who managed to reach overweight (14\%) and obesity (6\%) among patients with CD. ${ }^{14)}$
Short stature was seen in 32 patients (52.2\%) in the present study. Short stature was documented in $81.4 \%$ Turkish patients. ${ }^{2)}$ Yet, another study from Turkey found only 16.6\% to be short. ${ }^{1)}$ Short stature was also reported in 22\%, 20.9\%, and $17 \%$ of children with CD from Saudi Arabia, India, and Kuwait; respectively. ${ }^{3,10,14)}$ Like our study, a case-control study from Germany did not show a significant difference in height between $\mathrm{CD}$ seropositive and seronegative children despite that seropositive children tended to be shorter. ${ }^{6}$

$\mathrm{CD}$ is associated with many immune and some nonimmune related diseases. A variety of endocrine abnormalities have been reported to accompany or precede CD diagnosis. ${ }^{1,2)}$ Patients with insulin-dependent diabetes mellitus, thyroid disorders, Addison diseases, pernicious anemia, autoimmune thrombocytopenia, sarcoidosis, alopecia, and cardiomyopathies have higher risk of $\mathrm{CD}$ than normal population. ${ }^{1,5)}$ In the present study, associated conditions were found in $63.1 \%$ (41 of 65). History of food allergy was found in $25.4 \%$ (16 of 63). Likewise, Finnish study also reported concomitant chronic diseases in 61\% (315 of 517) and food allergy in $24.1 \%$ (125 of 518). ${ }^{15)}$ Yet, an American study found only $0.97 \%$ ( 4 of 411 ) of patients to have associated food allergies. ${ }^{11)}$

Type 1 diabetes mellitus commonly precedes $\mathrm{CD}$ diagnosis (10\%-25\% of cases) and it is more common in patients with CD compared to general population. ${ }^{1,2)}$ In this study, type 1 diabetes mellitus was seen in $13.8 \%$ (9 of 65) which is similar to the American study (12.8\%, 10 of 78). ${ }^{11)}$ Patients from Saudi Arabia and Oman had higher percentage of associated diabetes of $26.5 \%$ (30 of 113) and 22.2\% (2 of 9), respectively. ${ }^{3,7}$ ) However, studies from Turkey and Finland showed lower percentages of $6.4 \%$ (9 of 140 ) and $7.3 \%$ (41 of 516), respectively. ${ }^{2,15)}$

Nonspecific laboratory changes were documented in patients with $\mathrm{CD}^{2)}$ In the present study, low hemoglobin was noted in $51.8 \%$. Anemia was the most dominant laboratory feature in patients with CD from Turkey (56.4\%), India (100\%), and Kuwait. ${ }^{2,10,14)}$ Yet, only $37.4 \%$ of patients from Finland and $33.3 \%$ of patients from Mexico were anemic. ${ }^{15,18)}$ More specifically, IDA was seen in 23 patients (69.7\%) in our study. This is double than the prevalence of IDA in children aged 3 years from Bahrain (30\%). ${ }^{31)}$ Similar to our study, studies from India and Turkey also reported IDA in $60.4 \%$ and $45.7 \%$ respectively while a study from Mexico showed lower percentage (25\%). ${ }^{2,10,18)}$ In this study, IDA was diagnosed based mainly on low serum iron levels with or without low serum ferritin levels. However, all the patients tested for serum ferritin levels had normal values. This finding is expected because serum ferritin is an acute-phase reactant and can be raised in patients with infections, chronic diseases, or chronic inflammation. ${ }^{25}$ )

Elevated liver enzymes were noticed in 13 patients (30.9\%) in the current study with high ALT in 10 patients (23.8\%). CD-associated liver involvement has been noted by multiple studies; ranging between $34.8 \%$ to $38.8 \% .^{2,10,14,15)}$ Liver enzyme derangements are more frequently seen in symptomatic patients with $\mathrm{CD}$ and patients with Down syndrome, nephrotic 
syndrome and juvenile rheumatoid arthritis. ${ }^{10,14,15)}$

Autoantibody testing made a significant breakthrough in diagnosing both symptomatic and asymptomatic CD. ${ }^{6}$ More than $80 \%$ of patients with CD from Italy were diagnosed by both positive serology and histology. ${ }^{12)}$ In this study, the overall autoantibody tests were positive in 32 (71.1\%) out of 45 patients (67.2\%) with available data (AGA IgG in 64.1\%, AGA IgA in $48.4 \%$, AEA IgA in 44.8\%, and tTG IgA in 29\%). This is lower than values reported by other studies. ${ }^{2,14,10,19)}$ These differences might be explained by the presence of high percentage of IgA deficient patients $(10.3 \%)$ in our study compared to other studies where IgA deficiency was found in only in the range between $0.3 \%$ and $6.3 \%$. 2,4,7,10,12,18)

HLA markers remain the strongest risk factor for development of CD. ${ }^{4}$ In the current study, 8 patients were tested for HLA class II types and all of them were positive (100\%). Likewise, studies from Turkey and Mexico showed an overall positive HLA class II types in $97.4 \%$ and $73.3 \%$ of patients, respectively. 1,18 )

In the present study, endoscopic data were found in 25 patients (37.3\%), 16 of them (64\%) had abnormal duodenal findings. In a study from Poland, variable disease extensions have been documented by endoscopy. ${ }^{5)}$ Both continuous and interrupted focal intestinal lesions have been found. ${ }^{5)}$ In untreated patients, continuous lesions extending from the proximal part of duodenum to jejunum are more likely. ${ }^{5)}$ A study from Turkey revealed that 2 out of 14 patients (14\%) had normal endoscopic findings and one had unclassified sprue. ${ }^{1)}$

Traditionally, intestinal biopsy and histological assessment have been mandatory criterion standard of CD diagnosis. ${ }^{1,18)}$ Histological findings have higher sensitivity and specificity than HLA-DQ2/DQ8 and tTG-IgA positivity combined. ${ }^{1)}$ Although duodenal biopsy is very important in CD diagnosis, symptomatic cases with very high tTGA levels, positive AEA, and the diseaserelated HLA types can be excluded from being biopsied. ${ }^{1,20)}$ In the current study, 35 patients (52.2\%) had duodenal histological reports available. Eighteen patients (51.4\%) had histological findings suggestive of CD (Marsh II or III). The majority of these patients (88.9\%, 16 of 18) had Marsh III. Other studies also had a majority of Marsh III. ${ }^{4,10,12,14)}$ Histological findings vary between patients. ${ }^{10)}$ In the current study, Marsh IIIc was the commonest type found in $22.8 \%$ (8 of 35 ) compared to type IIIa in $17.1 \%$ (6 of 35 ) and type IIIb in $5.7 \%$ ( 2 of 35$)$. This is close to percentage reported from Italy and United States of America where Marsh IIIc was found in 37.5\% (42 of 112) and $32 \%$ (132 of 411), respectively. ${ }^{4,11)}$ However, Marsh IIIb was the commonest in patients from India, Finland, and Iraq. ${ }^{10,15,19)}$

In the present study, Marsh 0 was found in 28.6\%, Marsh I in 20\%, and Marsh II in 5.7\%. A study from Italy reported Marsh 0 in 13.4\%, Marsh I in 9.8\%, and Marsh II 4.5\%.4) In the current study, Marsh 0 was higher than the Italian study which can be explained by the older age at presentation in their patients. ${ }^{4)}$ It is more common to observe advanced histopathological stages in older children. ${ }^{11)}$ Another explanation is that IEL in the present study were detected on hematoxylin and eosin-stained samples in $51.4 \%$ (18 of 35$)$. IEL is considered a vital clue to diagnosis. ${ }^{20)}$ Unfortunately, these cells could be missed due to lack of contrast especially when counts are low. ${ }^{20)}$ A more sensitive method is immune-histochemical staining for CD3 which can improve the diagnosis accuracy. This is shown in a prospective study on 159 patients where a difference of $12.6 \%$ was found between the 2 methods. ${ }^{20)}$ In the present study, immuno-histochemical staining for CD3 was tested in 3 patients and showed increase in IEL in all of them.

Up to this date, the most efficient treatment for CD is strict adherence to GFD. ${ }^{3,11)}$ Yet, $64.3 \%$ of patients in the current study showed poor adherence to GFD. Patients who were followed up 1 year after starting GFD reported better general health status and less gastrointestinal symptoms, but adherence declined by $29 \%$ and even further down with time. ${ }^{3,6)}$ The latter could be attributed to high cost and unavailability of trustworthy gluten-free products. ${ }^{3)}$ In addition, as patients grew older more temptations develop and the parents lose control over diet especially since patients become more adventurous with their diet options and more influenced by peer pressure. ${ }^{3,6)}$

In this study, patients with positive CD serology were significantly older in age $(P=0.025)$ compared to seronegative patients. This might be attributed to the longer exposure to glutencontaining diet. However, a study from India on 66 children with CD up to 18 years of age did not show a significant correlation between serology and advancing age. ${ }^{32}$ )

In the current study, more severe duodenal histology was noted in seropositive patients based on Marsh-Oberhuber classification $(P=0.002)$. This finding was also reported by other studies in which the severity of small intestinal damage on histology was correlated with tTG IgA levels. ${ }^{10,32,33)}$ A study from Iraq showed a significant reduction in the levels of CD-specific antibodies along with histological remission after GFD which also support our finding. ${ }^{19)}$

Similar to any other retrospective studies, this study was limited by missing some relevant data. Moreover, low sample size was another limitation that can be explained by the underestimation and underdiagnoses of the disease that is a well-documented issue in other studies. ${ }^{6,14)}$ In addition, despite that SMC is the only tertiary care center in Bahrain, this study remains a singlecenter study and its' results cannot be completely generalized to the whole population. However, this study is the first study from Bahrain focusing on the clinical and pathological characteristics of patients with $\mathrm{CD}$ and it can form a valuable source of information for any future studies tackling the same group of patients.

In conclusion, although CD is an underdiagnosed disease, this study revealed a significant rise in CD prevalence in the last decade. Clinical findings are widely variable with atypical presentations are likely to be faced. Pallor, FTT, and abdominal distension were the most frequent presentations. IDA and allergic diseases were the commonest associated diseases. Marsh IIIc is the commonest positive histological finding which indicates more severe disease. Most patients had poor adherence with GFD. Further studies are required to focus on the degree of 
public and medical staff awareness about $\mathrm{CD}$; and to find out the reasons behind poor adherence to GFD in this group of patients.

\section{Ethical statement}

This study was conducted in accordance with the principles of Helsinki Declaration and it was ethically approved by the secondary care medical research subcommittee, Salmaniya Medical Complex, Ministry of Health, Bahrain (IRB number: 04-04-2016).

\section{Conflicts of interest}

No potential conflict of interest relevant to this article was reported.

\section{References}

1. Çakir M, Baran M, Uçar F, Akbulut UE, Kaklikkaya N, Ersöz S. Accuracy of HLA-DQ genotyping in combination with IgA anti-tissue transglutaminase serology and a "scoring system" for the diagnosis of celiac disease in Turkish children. Turk J Pediatr 2014;56:347-53.

2. Ertekin V, Selimoglu MA, Altinkaynak S. Celiac disease in childhood: evaluation of 140 patients. Eurasian J Med 2009;41:154-7.

3. Alsarkhy A, Elmouzan MI, Elshazaly S, Alanazi A, Alghamdi S, Anil S, et al. Clinical characteristics of celiac disease and dietary adherence to gluten free diet among Saudi children. Pediatr Gastroenterol Hepatol Nutr 2015;18:23-9.

4. Lionetti E, Castellaneta S, Francavilla R, Pulvirenti A, Tonutti E, Amarri $S$, et al. Introduction of gluten, HLA status, and the risk of celiac disease in children. N Engl J Med 2014;371:1295-303.

5. Szaflarska-Poplawska A, Parzçcka M, Kuczyńska R. The range of lesions in the small intestine of children with celiac disease determined by capsule endoscopy. Adv Clin Exp Med 2014;23:785-90.

6. Laass MW, Schmitz R, Uhlig HH, Zimmer KP, Thamm M, Koletzko S. The prevalence of celiac disease in children and adolescents in Germany. Dtsch Arztebl Int 2015;122:553-60.

7. Dehghani SM, Ehsaei Z, Honar N, Javaherizadeh H. Frequency of celiac disease in children with chronic functional constipation in Shiraz-Iran. Middle East J Dig Dis 2015; 7:166-9.

8. Rubio-Tapia A, Ludvigsson JF, Brantner TL, Murray JA, Everhart JE. The prevalence of celiac disease in the United States. Am J Gastroenterol 2012;107:1538-44.

9. Al Hatlani MM. Prevalence of celiac disease among symptom-free children from the Eastern Province of Saudi Arabia. Saudi J Gastroenterol 2015;21:367-71.

10. Rawal P, Thapa BR, Nain CK, Prasad KK, Singh K. Changing spectrum of celiac disease in India. Iran J Pediatr 2010;20:459-65.

11. Tanpowpong P, Broder-Fingert S, Katz AJ, Camargo Jr CA. Age-related patterns in clinical presentations and gluten-related issues among children and adolescents with celiac disease. Clin Transl Gastroenterol 2012;3:e9.

12. Zingone F, West J, Auricchio R, Bevilacqua RM, Bile G, Borgheresi P, et al. Incidence and distribution of celiac disease in Campania (Italy): 20112013. United European Gastroenterol J 2015;3:182-9.

13. Zingone F, West J, Crooks CJ, Fleming KM, Timothy RC, Ciacci C, et al. Socioeconomic variation in the incidence of childhood coeliac disease in the UK. Arch Dis Child 2015;100:466-73.

14. Al-Qabandi W, Buhamrah E, Alabdulrazzaq D, Hamadi K, AlRefaee F. Celiac disease in children: is it a problem in Kuwait? Clin Exp Gastroenterol 2015;8:43-8.

15. Nurminen S, Kivela L, Taavela J, Huhtala H, Maki M, Kaukinen K, et al. Factors associated with growth disturbance at celiac disease diagnosis in children: a retrospective cohort study. BMC Gastroenterol 2015;15:125.

16. Shahramian I, Dehghani SM, Haghighat M, Noori NM, Teimouri A,
Sharafi E, et al. Serological evaluation of celiac disease in children with congenital heart defect; a case cohort study. Middle East J Dig Dis 2015; 7:98-103.

17. Al-Lawati TT, Al-Musawi HS. Celiac disease in Oman: a tertiary centre experience. Oman Med J 2013;28:70-2.

18. Cruz NS, Barca AMC, Valenzuela JGH. Celiac disease in children from the northwest of Mexico: clinical characteristics of 24 cases. Rev Gastroenterol Mex 2013;78:211-8.

19. Osman M, Taha M, Al Duboni G. Assessment of the response to glutenfree diet in an Iraqi population with coeliac disease. A histological and serological follow-up study. Arch Med Sci 2014;10:294-9.

20. Mubarak A, Wolters VM, Houwen RHJ, Kate FJWT. Immunohistochemical CD3 staining detects additional patients with celiac disease. World J Gastroenterol 2015;21:7553-7.

21. Abdulsultan A, Crooks CJ, Card T, Tata LJ, Fleming KM, West J. Causes of death in people with coeliac disease in England compared with the general population: a competing risk analysis. Gut 2015;24:1220-6.

22. Al-Toma A, Volta U, Auricchio R, Castillejo G, Sanders D, Cellier C, et al. European Society for the Study of Coeliac Disease (ESsCD) guideline for coeliac disease and other gluten-related disorders. United European Gastroenterol J 2019; 7:583-613.

23. Husby S, Koletzko S, Korponay-Szabó IR, Mearin ML, Phillips jjA, Shamir R, et al. European Society of Pediatric Gastroenterology, Hepatology, and Nutrition guidelines for the diagnosis of coeliac disease. J Pediatr Gastroenterol Nutr 2012;54:136-60.

24. Song SM, Kim Y, Oh SH, Kim KM. Nutritional status and growth in Korean children with Crohn's disease: a single-center study. Gut Liver 2014;8:500-7.

25. Bouri S, Martin J. Investigation of iron deficiency anaemia. Clin Med 2108; 18:242-4.

26. WHO Multicentre Growth Reference Study Group. WHO Child Growth Standards: Length/height-for-age, weight-for-age, weight-forlength, weight-for-height and body mass index-for-age: Methods and development. Geneva (Switzerland): World Health Organization, 2006: 312.

27. De Onis M, Onyango AW, Porghi E, Siyam A, Nishida C, Siekmann J. Development of a WHO growth references for school-aged children and adolescents. Bull World Health Organ 2007;85:660-7.

28. Singh P, Arora A, Strand TA, Leffler DA, Catassi C, Green PH, et al. Global prevalence of celiac disease: systematic review and meta-analysis. Clin Gastroenterol Hepatol 2018;16:823-36.

29. Popp A, Mäki M. Changing pattern of childhood celiac disease epidemiology: contributing factors. Front Pediatr 2019;7:357

30. Nardecchia S, Auricchio R, Discepolo V, Troncone R. Extraintestinal manifestations of coeliac disease in children: clinical features and mechanisms. Front Pediatr 2019;7:56.

31. Al-Haddad L, Al-Dubaib R, Al-Najem W, Al-Shaikh Z, Al-Ghawi A. Iron deficiency anemia among 3-year-old children and its management in primary health care in Bahrain. J Bahrain Med Soc 2017;29:35-40.

32. Meena DK, Akunuri S, Meena P, Bhramer A, Sharma SD, Gupta R. Tissue transglutaminase antibody and its association with duodenal biopsy in diagnosis of pediatric celiac disease. Pediatr Gastroenterol Hepatol Nutr 2019;22:350-7.

33. Smarrazzo A, Misak Z, Costa S, Mičetić-Turk D, Abu-Zekry M, Kansu A, et al. Diagnosis of celiac disease and applicability of ESPGHAN guidelines in Mediterranean countries: areal life prospective study. BMC Gastroenterol 2017;17:17.

How to cite this article: Hasan M Isa, Eman Farid, Jaafar J. Makhlooq, Afaf M. Mohamed, Jumana G. Al-Arayedh, Fawzeya A. Alahmed, Shima Medani. Celiac disease in children: Increasing prevalence and changing clinical presentations. Clin Exp Pediatr 2021;64:301-9. https://doi.org/10.3345/cep. 2020.00304 